Ann. Zootech., I980, 29 (4), 40I-407.

\title{
Le besoin en méthionine pendant la période de finition chez le poulet de chair
}

\author{
M. LARBIER et B. LECLERCQ \\ avec la collaboration technique de A. M. CHAGNEAU \\ Station de Recherches Avicoles, I.N.R.A., Tours, \\ I.N.R.A. Nouzilly 37380 Monnaie (France)
}

\begin{abstract}
Résumé
Des poulets de chair de sexe mâle ont reçu entre les âges de 4 et 7 semaines, 6 régimes expérimentaux. Cinq d'entre eux titraient 18,3 p. I oo de protéines brutes (mais + tourteau de soja) et étaient supplémentés avec $0 ; 0,04 ; 0,08 ; 0,12$ ou $0,16 \mathrm{p}$. Ioo de DL-méthionine. Ils ont été comparés à un régime témoin renfermant $20,1 \mathrm{p}$. ıoo de protéines et $0,46 \mathrm{p}$. Ioo de méthionine.

Aucune différence n'a pu être mise en évidence entre les lots pour la vitesse de croissance. Mais l'utilisation des régimes à 18,3 p. Ioo de protéines non supplémentés avec de la DL-méthionine détériore l'efficacité alimentaire et conduit à la production d'animaux plus gras.

La supplémentation avec de la DL-méthionine améliore peu l'efficacité alimentaire mais réduit l'engraissement.
\end{abstract}

\section{Introduction}

Dans un régime alimentaire à base de maïs-tourteau de soja destiné au Poulet de chair, la méthionine est souvent considérée comme premier facteur limitant. Cependant, les études relatives à la détermination précise du besoin en cet acide aminé pour la période de finition (entre 4 semaines d'âge et le jour d'abattage) ne sont par concordantes. Dans le coût alimentaire de l'élevage du Poulet de chair, l'aliment de finition représente une part prépondérante, les trois quarts de la quantité totale d'aliment nécessaire étant consommés sous cette forme.

Dans les tables de recommandations les valeurs représentant le besoin, généralement majoré de $5 \mathrm{p}$. I oo pour tenir compte de la diversité qualitative des matières premières et de la disponibilité de la méthionine, sont très variables. Pour un aliment apportant entre 3 Ioo et $3200 \mathrm{Kcal} / \mathrm{kg}$ d'énergie métabolisable, les valeurs préconisées sont comprises entre 0,32 et 0,46 p. Ioo de l'aliment : 0,35 
(I.N.R.A. I974), 0,38 (ScoTr, I979), o,40 (N.R.C., I97I), o,4I (YounG et al., I976), 0,42 (A.E.C. I978), 0,44 (Thomas, Twinning et Bossard, r975), o,46 (Thomas et al., I978).

Dans les tables du S.I. (Spelderholt Institut), JANSSEN et al. (I979), d'une part associent les besoins en méthionine et cystine, d'autre part adoptent deux modes d'expression : l'un en teneur totale, l'autre en teneur d'acides aminés soufrés digestibles. Les valeurs recommandées sont respectivement 0,78 et $0,67 \mathrm{p}$. roo.

L'utilisation métabolique d'un acide aminé dépend non seulement de la densité énergétique de la ration mais aussi de la présence et du niveau d'apport des autres acides aminés. Dans le cas de la méthionine, les recommandations supposent que l'aliment finition titre $3200 \mathrm{Kcal}$ d'énergie métabolisable par $\mathrm{kg}$. Selon McGinnis et QUEST (I979), le rapport calories/protéines devrait être au maximum égal à ${ }^{6} 65$ soit une teneur en protéines minimum de 19,4 p. Ioo pour un taux énergétique de $3200 \mathrm{Kcal} / \mathrm{kg}$. Or, les résultats obtenus dans plusieurs expériences réalisées dans notre laboratoire, montrent qu'il est possible de réduire l'apport de protéines à $\mathrm{I} 8 \mathrm{p}$. Ioo sans modifier notablement les performances zootechniques (BLUM, I975). Après avoir défini le besoin en lysine (LARBIER et DUPONT, r980), nous nous proposons dans la présente expérience de déterminer le besoin en méthionine en prenant en considération à la fois les performances de croissance, l'efficacité alimentaire et l'état d'engraissement des poulets.

\section{Matériels et méthodes}

Deux mille huit cent quatre vingt poussins mâles issus d'un croisement commercial de type chair (Hubbard, France) sont élevés au sol dans 24 cases de $6 \mathrm{~m}^{2}$ chacune et soumis à un éclairement artificiel de 22 heures de lumière par nycthémère. Pendant les quatre premières semaines, ils reçoivent un aliment de démarrage granulé de type pratique (maïs-tourteau de soja) couvrant largement leurs besoins et dont la composition figure au tableau $\mathrm{I}$. Ce régime apporte 3 I20 Kcal d'énergie métabolisable par $\mathrm{kg}$ et renferme $22, \mathrm{I}$ p. Ioo de matières azotées totales $(\mathrm{N} \times 6,25)$. Les teneurs en lysine, méthionine et méthionine + cystine déterminées par analyse sont respectivement de 1,$23 ; 0,55$ et $0,94 \mathrm{p}$. Ioo.

A l'âge de 29 jours, les animaux sont pesés individuellement après une nuit de jeûne total. On constitue alors 6 lots comprenant chacun 480 poulets répartis en 8 répétitions. Du $29^{\mathrm{e}}$ au $49^{\mathrm{e}}$ jour, on distribue six régimes de finition granulés (diamètre $2,5 \mathrm{~mm}$ ) et isoénergétiques (3 I 7o Kcal d'énergie métabolisable par $\mathrm{kg}$ ). Le régime témoin renferme $20, \mathrm{I} \mathrm{p}$. Ioo de matières azotées totales $(\mathrm{N} \times 6,25)$, I, Io p. Ioo de lysine, 0,46 p. Ioo de méthionine et 0,79 p. roo de méthionine + cystine.

Les cinq autres régimes apportent les mêmes quantités de protéines (I $8,3 \mathrm{p}$. Ioo de matières azotées totales) et de lysine $(0,98 \mathrm{p}$. Ioo). Ils sont constitués à partir d'un régime de base renfermant $0,30 \mathrm{p}$. roo de méthionine et $0,63 \mathrm{p}$. Ioo de méthionine - cystine et supplémenté avec des doses croissantes de DL-méthionine $(0 ; 0,04 ; 0,08 ; 0,12 ; 0,16$ p. I00).

Dans ces aliments expérimentaux, les teneurs en méthionine et en méthionine + cystine varient respectivement de 0,30 à $0,46 \mathrm{p}$. Ioo et de 0,63 à $0,79 \mathrm{p}$. Ioo.

Le $49^{\mathrm{e}}$ jour, tous les animaux sont pesés individuellement après une nuit de jeûne total et la consommation alimentaite de chaque répétition est enregistrée. L'influence de la supplémentation en méthionine sur l'engraissement des poulets 
TABLEAU I

Composition des régimes expérimentaux

Composition of experimental diets

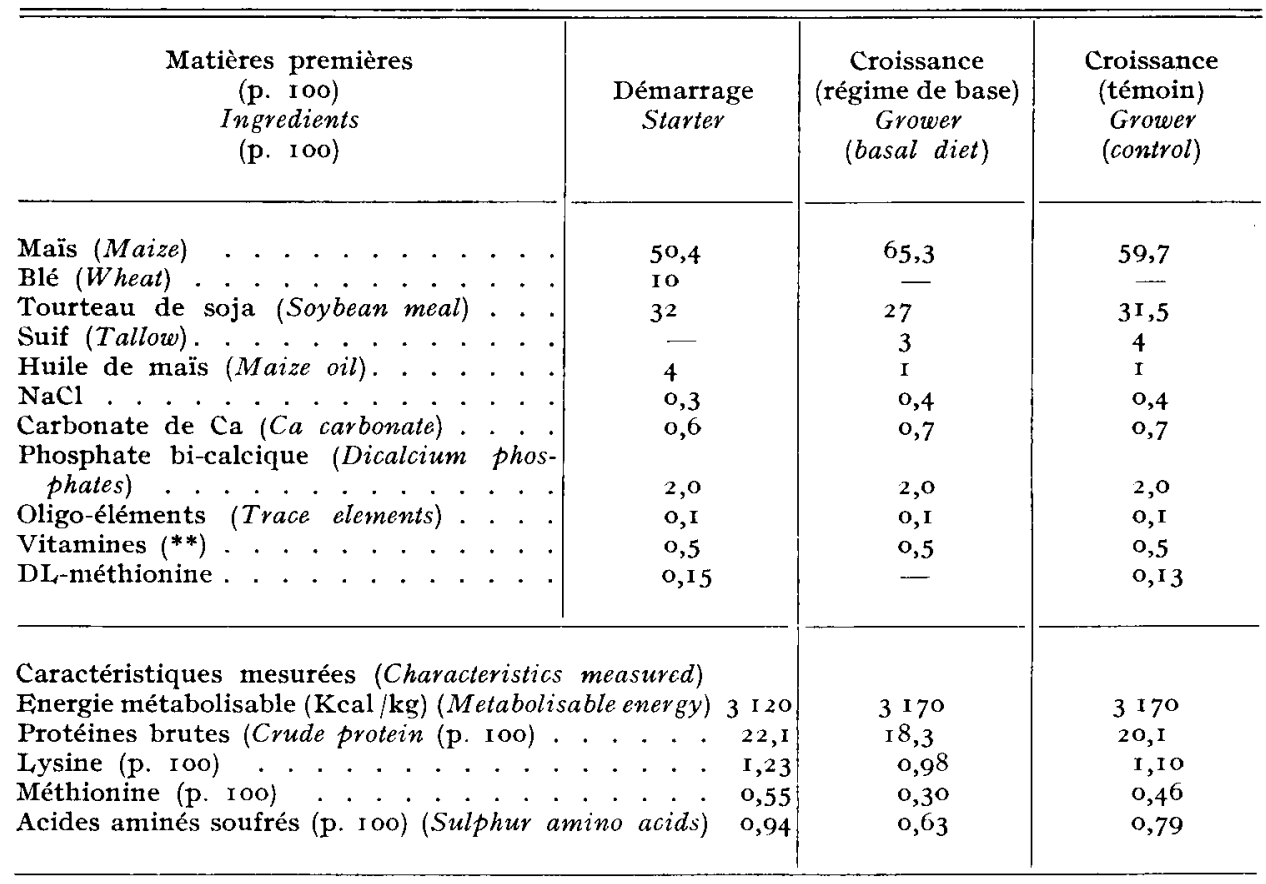

(*) Apportant en $\mathrm{mg}$ pour roo $\mathrm{kg}$ d'aliment : Co : 88; $\mathrm{Cu}: 875 ; \mathrm{I}:$ I28; Se : I5; $\mathrm{Zn}:$ Io ooo; Fe : $3500 ;$ Mn : I I ooo.

(**) Apportant pour roo kg d'aliment: Vit A : I ooo ooo UI; Vit $\mathrm{D}_{3}:$ I 50 ooo UI; Vit E: I, 5 g; BHT: I 2,5 g; Ménadione : 0,5 g; Riboflavine : 0,4 g; Pantothenate de Ca: $0,8 \mathrm{~g}$; Niacine : 2,5 g; Pyridoxine: $0,1 \mathrm{~g}$; Vit $\mathrm{B}_{12}: 0,8 \mathrm{mg}$; acide folique $: 0,02 \mathrm{~g}$; Biotine : $0,0 \mathrm{I} \mathrm{g}$; Chlorure de choline : $50 \mathrm{~g}$.

est étudiée chez des animaux issus du lot témoin et de deux lots expérimentaux recevant des régimes aux taux de méthionine extrêmes 0,30 et $0,46 \mathrm{p}$. Ioo. Vingt animaux par lot sont sacrifiés après une nuit de jeûne et la graisse abdominale est prélevée et pesée suivant la méthode de DELPECH et RICARD (I965).

\section{Résultats}

A la fin de la période de démarrage, la valeur moyenne du poids vif pour l'ensemble des animaux est de $890 \mathrm{~g}$. L'indice de consommation calculé en tenant compte du poids des poussins à la naissance est de $\mathrm{r}, 60$.

Quelle que soit la composition de l'aliment, les poids vifs réalisés, au $49^{\mathrm{e}}$ jour dans tous les lots ne sont pas significativement différents. Ils varient entre 2084 et 2 I $08 \mathrm{~g}$. En revanche, l'indice de consommation est significativement plus faible chez les animaux qui reçoivent le régime témoin titrant 20,1 p. Ioo de matières 


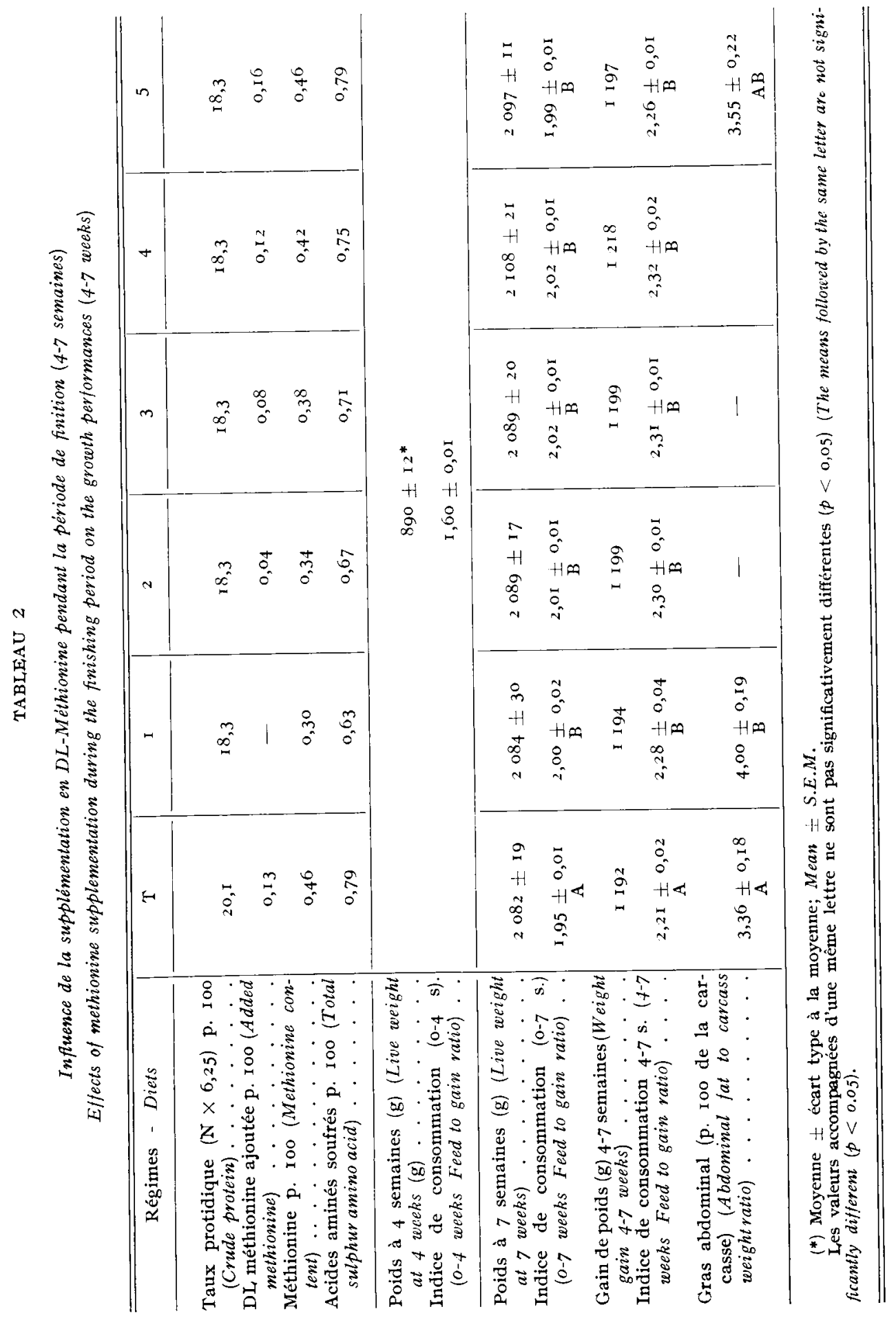


azotées et $0,46 \mathrm{p}$. I oo de méthionine. Les valeurs obtenues dans les cinq lots expérimentaux oscillent entre 2,26 et 2,32 et ne dépendent pas du taux de méthionine. Il en résulte un indice de consommation cumulé correspondant à l'ensemble des deux périodes d'élevage (o à 7 semaines) égal à $I, 95$ pour le lot témoin et compris entre 1,99 et 2,02 pour les autres lots.

Il n'en est pas de même pour l'engraissement des poulets. La quantité de graisse abdominale dépend du taux de méthionine, l'influence du taux protidique étant négligeable. Chez les animaux du lot témoin, la graisse abdominale représente $3,36 \mathrm{p}$. Ioo du poids de la carcasse. Cette proportion n'est pas significativement différente de celle $(3,55)$ observée chez les animaux nourris avec un aliment renfermant $I 8,3 \mathrm{p}$. Ioo de matières azotées totales mais apportant $1 \mathrm{a}$ même quantité de méthionine que le régime témoin $(0,46 \mathrm{p}$. Ioo). Elle est en revanche significativement plus faible que la valeur $(4,00 \mathrm{p}$. I 00$)$ observée lorsque l'aliment ne contient que $0,30 \mathrm{p}$. roo de méthionine et $0,63 \mathrm{p}$. roo de méthionine + cystine.

\section{Discussion}

Les performances zootechniques obtenues lorsque les animaux sont nourris avec le régime témoin ou le régime de base sont pour le croisement utilisé, tout à fait comparables à celles que l'on peut observer dans les conditions d'un élevage industriel. Le développement corporel est suffisant pour envisager l'abattage des animaux et leur commercialisation dès l'âge de 7 semaines.

Dans la pratique, les régimes de finition renferment souvent entre 20 et $22 \mathrm{p}$. I oo de matières azotées totales. Dans cet essai, la distribution d'aliment à taux protidique modéré ( $18,3 \mathrm{p}$. Ioo) n'exerce aucun effet dépressif sur la croissance mais diminue légèrement 1'efficacité alimentaire. De nouveau nous vérifions qu'il est possible de réduire la proportion de tourteau de soja dans le régime en augmentant celle des céréales (BLUM, I975; LARBIER et DUPONT, I980).

En utilisant des régimes titrant 18,3 p. Ioo de protéines, nous montrons que le besoin en méthionine du Poulet de chair pendant la période de finition (entre 4 et 7 semaines) n'excède pas $0,30 \mathrm{p}$. I 00 correspondant à un apport total d'acides aminés soufrés égal à 0,63 p. Ioo. Dans notre essai, ni le développement corporel, ni l'indice de consommation ne sont améliorés par la seule supplémentation en méthionine. Dans les expériences de PICARD (A.E.C., I974), les animaux reçoivent comme dans la présente étude, une succession de deux aliments renfermant 20 et $\mathrm{I} 8 \mathrm{p}$. Ioo de matières azotées totales. Les performances zootechniques sont légèrement améliorées en augmentant de 0,62 à $0,80 \mathrm{p}$. Ioo la teneur en acides aminés soufrés. Mais, ces effets pourraient ne pas être liés à la seule addition de DL,-méthionine, car dans le même temps, l'apport alimentaire de lysine est passé de 0,93 à $I, 02$ p. xoo.

En ne tenant compte que du gain de poids, nos résultats sont à rapprocher de cetux de SEKIZ et al. (I975). Utilisant un régime semi-synthétique, ces auteurs ont obtenu une croissance maximum lorsque l'apport alimentaire de méthionine est de 0,32 p. Ioo. Mais, en supplémentant le régime avec de la DL-méthionine, ils ont enregistré un indice de consommation minimum lorsque la teneur totale en cet acide aminé est de $0,46 \mathrm{p}$. Ioo.

Dans les travaux de ELDRED et al. (1974), un seul aliment est distribué depuis la naissance, 1'apport de méthionine variant de 0,34 à $0,54 \mathrm{p}$. Ioo. Les perfor- 
mances de croissance et d'efficacité alimentaire sont enregistrées à 42 et 56 jours respectivement pour les mâles et les femelles. Les meilleurs résultats sont obtenus lorsque le taux de méthionine est de $0,46 \mathrm{p}$. roo, pour les poulets mâles et $0,42 \mathrm{p}$. roo pour les femelles. Mais ces conditions expérimentales inhabituelles ne permettent pas de distinguer sur le plan nutritionnel les deux périodes de démarrage et de finition : le besoin en acides aminés exprimé en $p$. Ioo de la ration diminuant régulièrement pendant la croissance (BLUM, I975).

Parallèlement au gain de poids et à l'efficacité alimentaire, l'état d'engraissement, critère de qualité du poulet, peut être pris en compte dans la détermination des besoins en énergie et en protéines (GUILIAUME, I975). Nous montrons que chez des poulets de même origine génétique à âge, poids vif et indice de consommation égaux, la quantité de graisse abdominale est variable et dépend du taux de méthionine dans l'aliment (Prasad \& SAdagopan, I976).

La relation entre l'engraissement, la quantité d'acides aminés ingérée et la densité énergétique de la ration a fait l'objet de nombreux travaux revus récemment par NELSON (1980). Aux taux protidiques élevés, les différences de composition de la carcasse sont généralement très faibles. En revanche, la distribution d'un aliment ayant un taux protidique bas augmente la proportion de graisse abdominale (LIPSTEIN et al., I975; KORELESKI \& Rys, I979). Cet effet serait dû à la surconsommation alimentaire liée à une subcarence en acides aminés indispensables (SEkrz et al., I975). Dans notre essai, nous vérifions que la diminution du taux protidique augmente à la fois la consommation alimentaire et la quantité de graisse abdominale. Mais nous montrons aussi que la seule supplémentation en DLméthionine diminue l'engraissement sans modifier significativement le niveau d'ingestion de l'aliment.

En définitive, si l'on ne considère que les strictes performances de croissance, les besoins en méthionine et en méthionine + cystine pendant la période de finition ne dépassent pas respectivement 0,30 et $0,63 \mathrm{p}$. roo. Mais dans la mesure où l'on tient compte de l'engraissement dont l'excès peut être préjudiciable à la qualité de la carcasse, la supplémentation du régime de type maīs-tourteau de soja ayant un taux protidique modéré (I $8,3 \mathrm{p}$. 100) avec de la DL-méthionine s'avère nécessaire.

Accepté pour publication en octobre I980.

\title{
Summary
}

\author{
Methionine requirement of broiler chickens \\ during the finishing period
}

Male broiler chickens were fed 6 experimental diets between 4 and 7 weeks of age. Five of these diets included 18.3 p. Ioo crude protein (maize + soybean meal) and were supplemented with $0 ; 0.4,0.08,0.12$ or 0.16 p. Ioo DL-methionine. They were compared with a control diet containing $20 . \mathrm{I}$ p. Ioo protein and 0.46 methionine.

There was no difference in the growth rate of the various groups of animals. However, utilisation of 18.3 p. roo protein diets without any DL-methionine supplementation reduced the feed efficiency and led to production of fatter animals.

DL-methionine supplémentation improved only little the feed efficiency, but reduced the fatness of the animals. 


\section{Références bibliographiques}

A.E.C., 1974. Taux protidique bas. Inf. volailles, no 240 (A.E.C. 03600 Commentry, France).

A.E.C., 1978. Alimentation animale: énergie, acides aminés, vitamines et minéraux. Doc. no 4 (A.E.C., o360o Commentry, France).

Bı,UM J. C., I975. Les apports azotés dans 1'alimentation du Poulet de chair. In Les volailles de consommation (Station de Recherches Avicoles, Nouzilly, 37380 Monnaie, France).

DELPECH P. RICARD F. H., I965. Relation entre les dépôts adipeux viscéraux et les lipides corporels chez le poulet. Ann. Zootech., 14, I8I-I 89.

EldDRED A. R., DAmRon B. L., HARMS R. H., I974. Sulfur amino acid requirement of male and female broilers. Poult. Sci., 53, I921.

Guillaume J., I975. Les apports énergétiques dans l'alimentation du Poulet de chair. In Les volailles de consommation (Station de Recherches Avicoles, Nouzilly, 37380 Monnaie, France).

I.N.R.A., I974. L'alimentation du Poulet de chair. Station de Recherches Avicoles, Nouzilly, 37380 Monnaie, France.

Janssen W. M. M., TERPstra K., BeEking F. F. E., Bisalsky A. J. N., 1979. Feeding values for poultry. Spelderholt Institute for Poultry Research, Beekbergen, Pays-Bas.

KORELESKI J., RYS R., I979. Effect of reduced dietary protein and amino acid levels on the performance of broiler chickens. Feedsiu/fs, 51 (38), 39-42.

IAARBIER M., Duponx C., I980. Réduction du taux protidique des aliments poulet de chair et efficacité de la L-1ysine, HCl. VIe Congrès Europ. d'Aviculture. Hambourg, 3, 264-27 I.

LIPSTEIN B., BORNSTEIN S., BARTOV I., 1975. The replacement of some of the soybean meal by the first limiting amino acid in practical broiler diets. 3. Effects of protein concentration and amino acid supplementations in broiler finisher diets on fat deposition in the carcass. Br. Poult. Sci., 16, 627.

Mc Ginnis C. H. Jr, QUest R., I979. Practical amino acid recommandation for broilers. Feedstuffs, 51, 24-25.

NATrONAL, RESEARCH COUNCIL, I97I. Nutrient requirement of Poultry. (Nat. Acad. Sci., Washinghton, D.C.).

Nelson T. S., I980. Feeding for changes in body composition of broilers. Proc. the Florida Nutrition Conference, I59-I 72.

Prasad A., Sadagopan V. R., I976. Effect of supplemental methionine in broiler diets on dressing percentage and carcass composition. Indian $J$ Nutr. Dietetics, 13, 406-410.

Scot'T M. L., I979. Nutrient requirements of chickens, turkeys. Feedstuffs, 51 (29), 63-64.

Sekiz S. S., ScotT M. L., Nesheim M. C., I975. The effect of methionine deficiency on body weight, food and energy utilization in the chick. Poult. Sci., 54, I184-1 I 88 .

Thomas O. P., Twinning P. V. Jr, Bossard E. H., I975. The amino acid and protein requirements for broilers. Proc. Maryland Nutr. Conf., 48-53.

Thomas O. P., Twinning P. V., Bossard E. H., Nicholson J. L., I978. Updated amino acid requirements of broilers. Proc. Maryland Nutr. Conf., I o7-I I I.

Young R. J., Scotr M. L., AUstic R. E., Combes G. F. Jr, I976. A review of the nutrient requirements for chicks pullets and laying hens. Proc. Cornell Nutr. Conf., 45-58. 